\title{
Performance and stability of near-unity aspect ratio plasmas in the Pegasus Toroidal Experiment ${ }^{a}$ )
}

\author{
G. D. Garstka, ${ }^{\text {b) }}$ S. J. Diem, R. J. Fonck, B. T. Lewicki, A. C. Sontag, K. L. Tritz, \\ and E. A. Unterberg \\ University of Wisconsin-Madison, 1500 Engineering Drive, Madison, Wisconsin 53706
}

(Received 15 November 2002; accepted 16 January 2003)

\begin{abstract}
The Pegasus Toroidal Experiment [R. Fonck et al., Bull. Am. Phys. Soc. 41, 1400 (1996)] is a spherical torus designed to study the limits of plasma behavior as the aspect ratio $A$ approaches unity. Access to near-unity $A$ is achieved through the use of a novel high-stress reinforced solenoid magnet. High toroidal beta $\beta_{t}$ is obtained in ohmically-heated plasmas by operation at low field with densities up to the Greenwald limit. Values of $\beta_{t}$ up to $20 \%$ and normalized beta up to 5 have been obtained. The ratio of plasma current to toroidal field rod current, known as the toroidal field utilization, reaches values as large as 1 but appears to approach a "soft" boundary at that level related to both ohmic flux limitations and the onset of resistive magnetohydrodynamic (MHD) activity. The $m / n=2 / 1$ and $3 / 2$ modes are most frequently observed, in agreement with the inferred safety factor profiles. Experiments are beginning to access the external kink stability boundary at edge safety factor $q_{95}=5$, which is significantly higher than that observed in conventional tokamaks. Calculations using the DCON code [A. H. Glasser and M. S. Chance, Bull. Am. Phys. Soc. 42, 1848 (1997)] confirm instability to the ideal kink. (C) 2003 American Institute of Physics.
\end{abstract}

[DOI: $10.1063 / 1.1559972]$

\section{INTRODUCTION}

The spherical torus, or ST, is a toroidal confinement concept where the aspect ratio $A$ is less than 2 . The ST is an evolutionary extension of the tokamak concept, and has features that make it attractive compared to the tokamak. ${ }^{1}$ These features include compactness, high "natural" elongation, high bootstrap fraction with good alignment, high stable toroidal beta $\left(\beta_{t}=\langle n T\rangle / B_{t 0, v a c}^{2}\right)$, and high toroidal field utilization, which here is defined as the ratio of the plasma current $\left(I_{p}\right)$ to the toroidal field rod current $\left(I_{t f}\right)$. Balancing the advantages of the ST are distinct disadvantages, which stem primarily from the small size of the center column. All of the centerstack components of a tokamak-toroidal field coils, ohmic solenoid, diagnostics, plasma-facing components, cooling - must be engineered and built into a small radius for the ST. This makes current drive by ohmic induction increasingly untenable for the ST, and would force a "burning" ST to use normally conducting toroidal field coils.

The balance between these advantages and disadvantages becomes particularly pointed as the aspect ratio is reduced to near unity. The Troyon limit on normalized beta ${ }^{2}$ $\left(\beta_{N}=\beta_{t} / I_{N}\right.$, where $\left.I_{N}=I_{p} / a B\right)$ increases dramatically as $A$ approaches 1 , and $I_{p} / I_{t f}$ can become significantly greater than 1 without destabilizing the external kink. The external kink boundary itself may change as $A$ approaches 1 , with unstable values of edge safety factor $q_{\psi}$ increasing above the conventional limits of $2-3.3,4$

The Pegasus Toroidal Experiment ${ }^{5}$ (Pegasus) is an extremely low aspect ratio ST developed to explore quasi-

\footnotetext{
a) Paper BI1 2, Bull. Am. Phys. Soc. 47, 19 (2002).

${ }^{b)}$ Invited speaker. Electronic mail: garstka@engr.wise.edu
}

spherical high-pressure plasmas with the goal of minimizing the central column while maintaining good confinement and stability. Pegasus is designed to expand the operational space of the ST to the region where $A<1.3$ and $I_{p} / I_{t f}>2$. This includes the study of magnetohydrodynamic (MHD) stability limits at very-low aspect ratio. It is also designed to explore the overlap with the spheromak at high toroidal field utilization. Figure 1 shows a contour of toroidal field utilization versus aspect ratio for fixed $q_{\psi}=6$ and fixed elongation $\kappa$ calculated using an analytic expression. ${ }^{1}$ The toroidal field utilization is essentially a measure of the toroidal field required for kink stability and is thus a metric of performance of the ST. Figure 1 also gives a rough illustration of the region of this space for several ST devices. Pegasus can be seen to cover the space between the low aspect ratio mainline ST experiments such as the Small Tight Aspect Ratio Tokamak (START), ${ }^{6}$ the National Spherical Torus Experiment (NSTX), ${ }^{7}$ and the Mega-Amp Spherical Tokamak (MAST) ${ }^{8}$ and the rod-stabilized spheromak experiments such as TS-3 $3^{9,10}$ and TS-4.

In this paper, initial results from the operation of the Pegasus Toroidal Experiment will be reported. Section II details the device and its operation. Section III describes general plasma results. Section IV discusses the observation and effects of MHD instabilities, including tearing modes and ideal kink modes. The final section summarizes the results and presents future directions for the project.

\section{EXPERIMENTAL APPARATUS}

The Pegasus Toroidal Experiment is an ultra-low aspect ratio ST. Figure 2 shows a cross-sectional drawing of the device. It features a major radius of $0.2-0.45 \mathrm{~m}$ and center- 


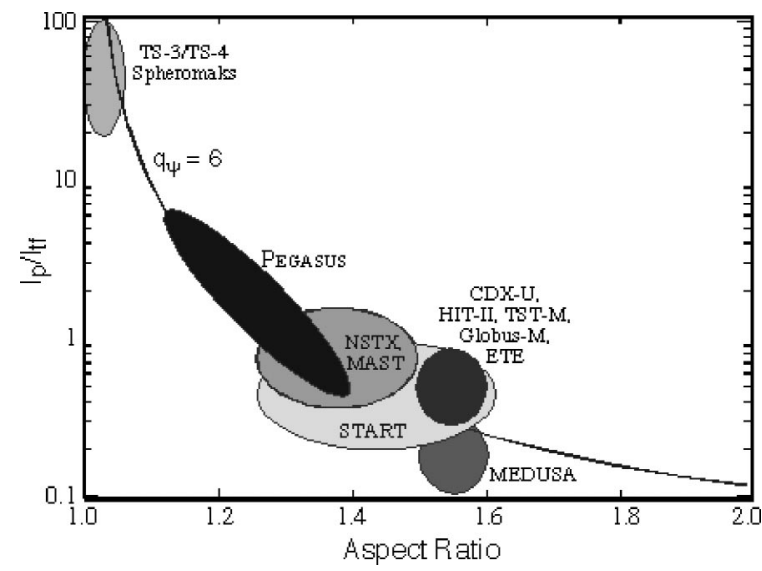

FIG. 1. Plot of toroidal field utilization vs aspect ratio for constant $q_{\psi}=6$ and for selected ST devices.

stack radius of $0.055 \mathrm{~m}$, which gives an aspect ratio $A$ $=1.13-1.3$. The toroidal field rod current can be as large as $0.15 \mathrm{MA}$, which corresponds to a vacuum field of 0.07 Tesla at $R=0.4 \mathrm{~m}$. Values of plasma current up to $0.16 \mathrm{MA}$ have been obtained. Typical discharge lengths range from 0.01 to $0.03 \mathrm{~s}$. All of the plasmas discussed in this paper are limited, and are produced by ohmic heating only.

The key to the operation of Pegasus as an ohmic ST is the high-stress solenoid, ${ }^{11}$ which was produced by the National High Magnetic Field Laboratory. The solenoid is capable of operation up to $20 \mathrm{~T}$ for 1000 pulses, at which level is produces $0.17 \mathrm{~Wb}$ flux swing for bipolar drive at $100 \mathrm{kA}$. It is routinely operated at $10 \mathrm{~T}$. The magnet consists of a double wrap of GlidCop Al 60, a high strength copper alloy, with 192 turns total. Outside each wrap is a reinforcing layer of carbon-fiber wrap, kapton insulation, and s-glass. The magnet is housed in a thin steel vacuum shell. The toroidal field center rods pass through the center of the solenoid mag-

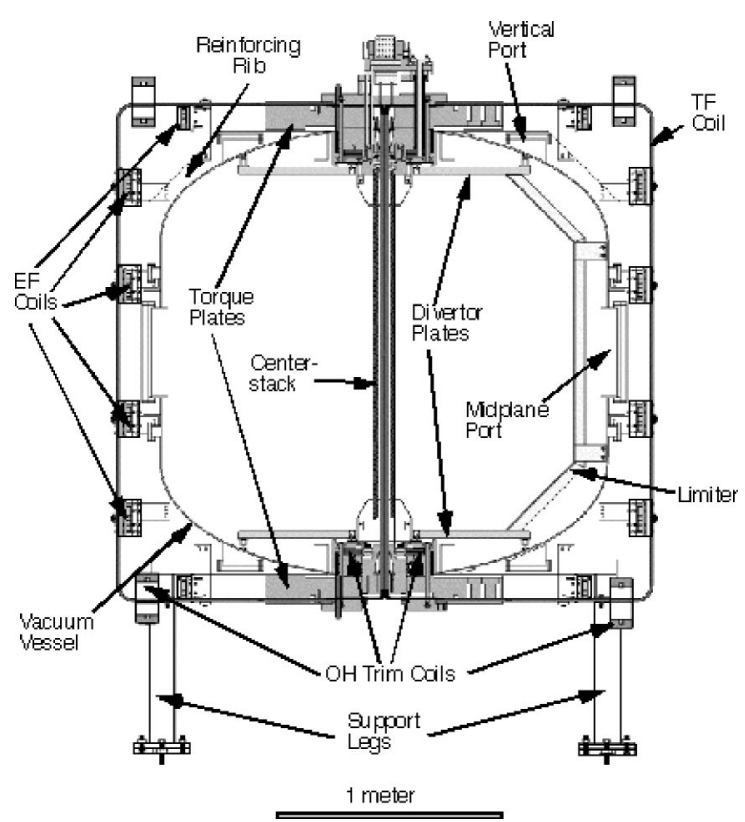

FIG. 2. Cross-sectional drawing of the Pegasus Toroidal Experiment.
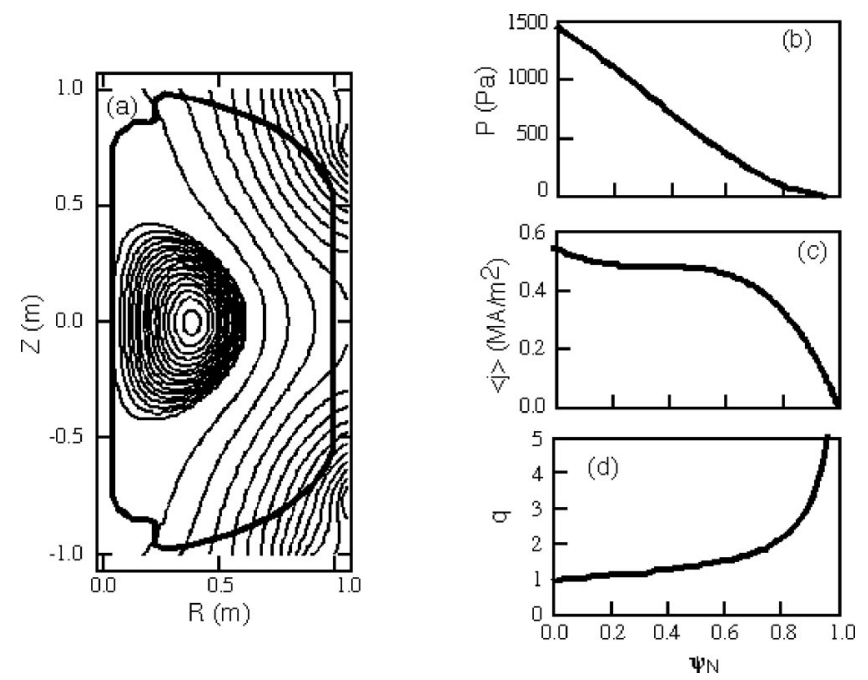

FIG. 3. Sample results from an MHD equilibrium reconstruction: (a) poloidal flux contours, (b) pressure vs normalized flux, (c) flux-surface-averaged current density vs normalized flux, and (d) safety factor vs normalized flux.

net, along with channels for water cooling. The entire assembly fits in a $0.1 \mathrm{~m}$ diameter. The solenoid magnet is run as a resonant circuit. Plasma forms when the loop voltage, following a half sine waveform, rises to approximately $3 \mathrm{~V}$.

The primary diagnostics used on Pegasus are magnetics. These include two internal plasma Rogowski coils and two internal diagmagnetic loops, 26 internal flux loops, including 6 behind the centerstack armor, 6 external flux loops to constrain wall currents, and 56 Mirnov coils which also function as B coils. The Mirnov coils are grouped into four arraystwo poloidal arrays (including centerstack coils) and two toroidal arrays, one on the centerstack and one on the low-field side. Other diagnostics include filterscopes, a 19-channel soft $\mathrm{x}$-ray array, a $270 \mathrm{GHz}$ microwave interferometer ${ }^{12}$ provided by the University of California-Los Angeles, a vacuum ultraviolet survey spectrometer, ${ }^{13}$ a fast imaging camera, and a 2D soft x-ray camera. ${ }^{14}$

Equilibrium reconstruction is the primary analysis tool for Pegasus plasmas. A new equilibrium code has been developed specifically for use on this device. This code is written to allow the incorporation of data from a wide range of diagnostics, but in particular measurements from the 2D soft $\mathrm{x}$-ray camera. The code performs a full solution of the GradShafranov equation at each iteration using a Gauss-Seidel multigrid relaxation technique on a $2 \mathrm{D}$ grid. Between iterations, the normalized error $\chi^{2}$ is minimized via the Levenberg-Marquardt technique; this then determines the starting point of the next iteration. Reconstructions usually employ polynomial parameterization for the pressure and $\mathrm{FF}^{\prime}$ profiles; typically two terms are used for the pressure profile and three terms for the $\mathrm{FF}^{\prime}$ profile. Uncertainties in the reconstructions are determined by a Monte Carlo analysis of the measurement errors. For discharges constrained by magnetics only, typical relative errors in major parameters are: $I_{p}=2 \%, q_{95}=6 \%, l_{i}=9 \%, R_{0}=4 \%$, and $\beta_{t}=15 \%$. Results from a sample equilibrium reconstruction for Pegasus are shown in Fig. 3. 


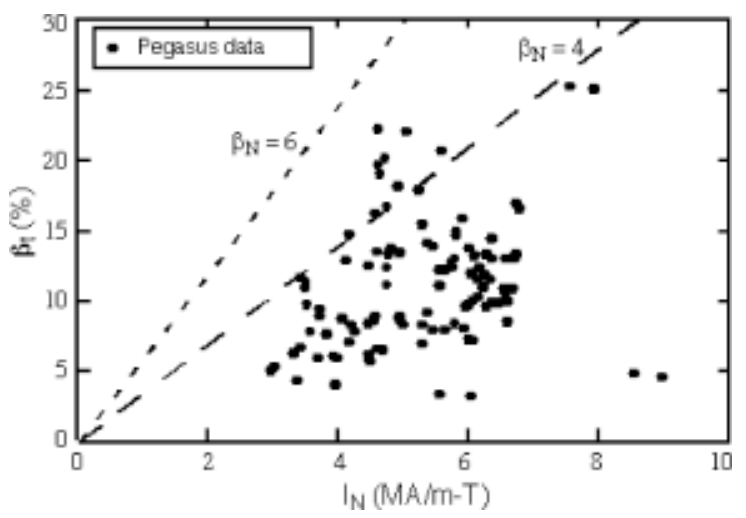

FIG. 4. Toroidal beta vs normalized current for selected Pegasus discharges. Contours of normalized beta $=4$ and 6 are indicated.

\section{PLASMA PERFORMANCE AT ULTRALOW ASPECT RATIO}

The Pegasus Toroidal Experiment has produced plasmas that demonstrate characteristics of ultralow-A operation. Normalized currents up to 6.5 have been observed, and values of $I_{p} / I_{t f}$ up to 1.1 have been routinely achieved. Values of $\beta_{t}$ up to $20 \%$ and $\beta_{N}$ up to 5 have been obtained ohmically with no evidence of beta limiting instabilities. A scatter plot of $\beta_{t}$ versus $I_{N}$ is given in Fig. 4. The Pegasus data shown in this plot significantly overlap data from the START experiment. ${ }^{15}$ These large values of $\beta_{t}$ are produced by operation at high densities (up to the Greenwald limit) and low applied toroidal field on axis, down to 0.04 Tesla. Typical values of $\beta_{p}$ and $\epsilon=1 / A$ are 0.3 and 0.85 , implying a paramagnetic regime. The paramagnetism in Pegasus is highly pronounced, with values of $R B_{t}$ up to $50 \%$ larger than the vacuum value at the magnetic axis. Transient values of $\kappa$ up to 3.7 have been observed. The internal inductance is low compared to standard tokamak plasmas. A scatter plot of $l_{i}$ versus $q_{95}$ for a number of Pegasus discharges is shown in Fig. 5. The low internal inductance is due to the high plasma current ramp rates (up to $30 \mathrm{MA} / \mathrm{s}$ ) and relatively short pulse length of these plasmas. The internal inductance typically relaxes on the current relaxation time, which is on the order of $0.03 \mathrm{~s}$. In these limited plasmas $q_{\text {lim }}$ is larger than $q_{95}$, but $q_{95}$ is used in this paper for comparison both with other ST devices and with diverted discharges that will be produced on Pegasus in the near future.

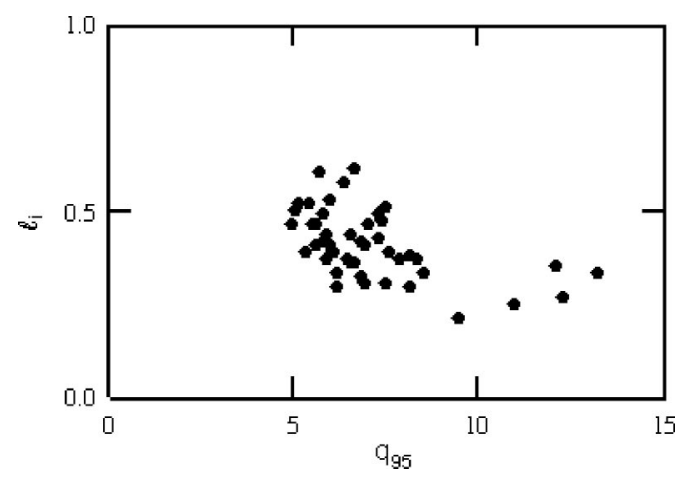

FIG. 5. Internal inductance vs $q_{95}$ for selected Pegasus discharges.

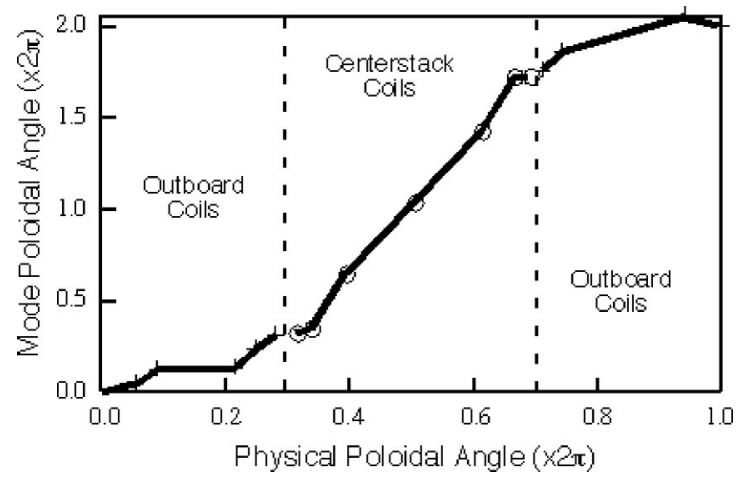

FIG. 6. Measured poloidal phase of $m / n=2 / 1$ mode vs poloidal angle of measuring coils. The measurements made by the centerstack coils and by the outboard coils are indicated.

The highly toroidal nature of this system can be seen in the structure of tearing modes. The large toroidal field gradient in an ST implies that the majority of the length of field lines on the outer flux surfaces is on the high-field side of the device. This characteristic can be directly observed by reconstructing the phase of magnetic islands observed by the poloidal Mirnov coils. Figure 6 gives the measured poloidal phase of an $m / n=2 / 1$ tearing mode versus the physical poloidal location of the coils. In this figure, $3 \pi$ out of $4 \pi$ of the total poloidal phase shift is observed on the center column Mirnov coils, which occupy only $2 \pi / 3$ of the $2 \pi$ poloidal extent of the coils. The measured amplitude of the tearing mode is another consequence of the high toroidicity of the system. The low-field-side coils observe a field perturbation of 4-6 Gauss at $r \approx 2 a$, which is comparable to or larger than the signal observed by the core coils, which are located at $r \approx 1.1 a$.

Another anticipated characteristic of very-low $A$ plasmas is that the magnetic shear is expected to be very low in the center, in contrast with the high edge shear. Initial measurements of the q-profile on Pegasus made by the 2D soft x-ray camera confirm this expectation. This camera measures the soft x-ray intensity across the plasma. Intensity contours are determined from which flux surfaces can be calculated. The flux surfaces are then used as an input to the equilibrium code, where they strongly constrain the safety factor profile. A q-profile determined by this technique is shown in Fig. 7, where the central shear can be seen to be near zero. In this case, the equilibrium profiles are modeled with a spline representation. The large error bars on this measurement are due to the long sampling time $(2 \mathrm{~ms})$ required by the soft $\mathrm{x}$-ray camera; these data "compete" with the instantaneous magnetics data and force larger error bars. These errors will be reduced when an upgraded version of the diagnostic is deployed in 2003.

As mentioned previously, values of the toroidal field utilization up to roughly 1 have been routinely achieved. However, a "soft limit" is found at $I_{p} / I_{t f} \approx 1$. This is illustrated in Fig. 8, which shows a plot of plasma current versus toroidal field rod current. This bound does not represent disruptive terminations or rapid drops in the plasma current. Rather, the plasma current is found to saturate or gently roll over at or 


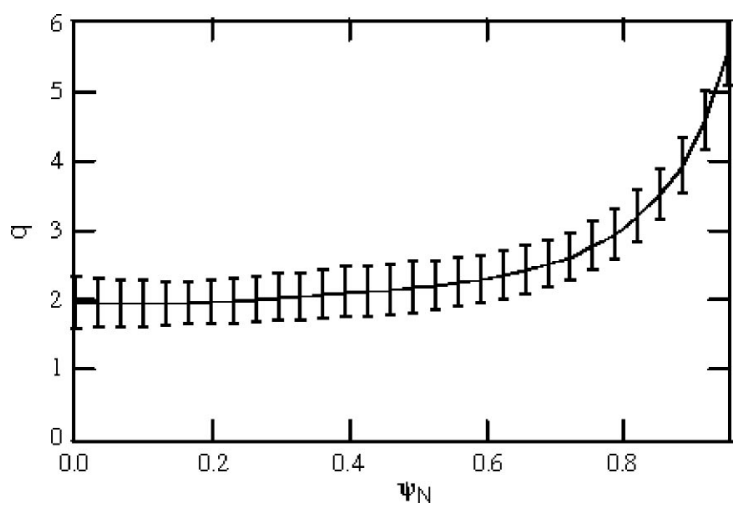

FIG. 7. Safety factor profile measured using soft $x$-ray measurements of flux surface shape as constraints on the equilibrium reconstruction.

below the level of $I_{t f}$. Two factors appear to contribute to this limit. The primary factor, to be discussed in the next section, is the presence of large-scale internal resistive MHD instabilities that degrade the plasma confinement. The second factor is related to plasma breakdown mechanics. As the toroidal field is reduced, the plasma breaks down later in time. This translates into a loss of volt-seconds available to the plasma. This effect is relatively minor, however, and cannot alone explain the drop in $I_{p}$ with $I_{t f}$.

\section{MHD INSTABILITIES}

\section{A. Internal resistive modes}

In almost all Pegasus discharges, significant rotating distortions in the poloidal field are observed by the Mirnov coils. These distortions, which rotate in the electron diamagnetic drift direction with a frequency between 2 and $10 \mathrm{kHz}$, are identified as low-order tearing modes. An example of this mode activity is given in Fig. 9. Figure 9(a) shows the plasma current versus time, while Fig. 9(b) shows a low-field side measurement of the fluctuating field. The most commonly observed mode is an $m / n=2 / 1$ mode, although $m / n$ $=3 / 2,3 / 1$, and $4 / 1$ have also been observed.

It is well known that tearing modes degrade plasma confinement and performance. ${ }^{16}$ These modes are particularly severe in Pegasus due to a combination of factors. First, Pegasus has a very low toroidal field and features a relatively

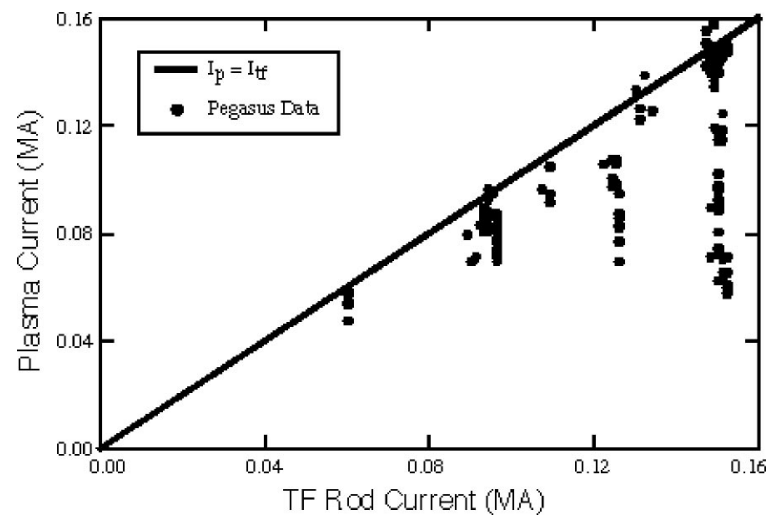

FIG. 8. Maximum plasma current plotted vs toroidal field rod current for selected discharges. The line $I_{p}=I_{t f}$ is also indicated.
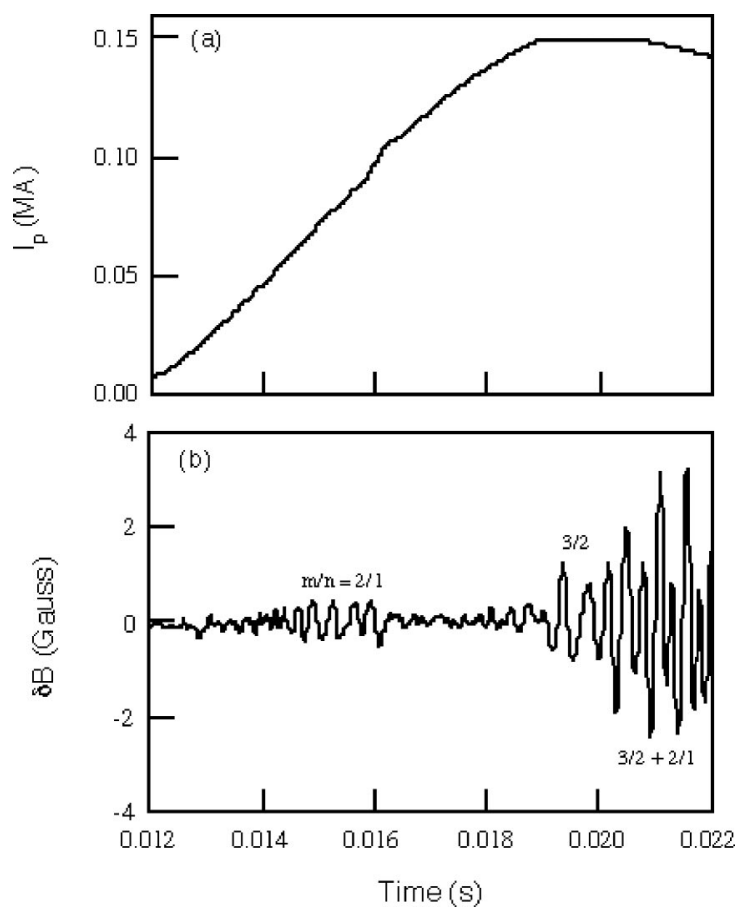

FIG. 9. Tearing mode signals vs time: (a) plasma current, (b) fluctuation signal from a low-field-side Mirnov coil. Cross-phase analysis of mode signals reveals the presence of $m / n=$ both $2 / 1$ and $3 / 2$ in this example.

fast current ramp. This implies that low-order rational flux surfaces appear early in the discharge evolution when the plasma is still fairly resistive. Second, safety factor profiles in Pegasus, and in STs generally, tend to have low shear in the center of the plasma. The first factor implies rapid growth of resistive modes in the nonlinear Rutherford regime. The second implies that the saturated island sizes, which in a cylindrical plasma should scale as $q(d q / d r)^{-1}$, are large. This prediction is borne out in measurements of the Pegasus magnetic islands. Soft x-ray measurements (in a relatively coarse array) find that the fluctuations encompass almost the entire plasma. Calculations of island size using Mirnov coil signals employing a conservative cylindrical approximation indicate that the saturated island widths are greater than 0.1 $\mathrm{m}$, and are on the order of the minor radius.

It is found that the normalized tearing mode activity becomes larger as the toroidal field is reduced. This is illustrated graphically in Fig. 10, where normalized tearing mode amplitude for plasmas along the $I_{p}=I_{t f}$ line is plotted versus the toroidal field current. At the highest levels of toroidal field, flux consumption analyses indicate that values of the Ejima coefficient ${ }^{17}$ can be as low as 0.4 , indicating good use of ohmic flux in plasma formation. The Ejima coefficient rises along the $I_{p}=I_{t f}$ contour as the toroidal field is reduced, indicating poorer use of the available flux. In addition, the plasma stored energy and confinement time decrease along the same contour.

The appearance of these modes can be correlated with the presence of low-order rational flux surfaces in the lowshear plasma center. Figure 11(a) shows the evolution of $q_{0}$ versus time on the left axis, and the amplitude of the $\mathrm{m} / \mathrm{n}$ $=2 / 1$ mode activity on the right axis. Figure 11(b) shows the 


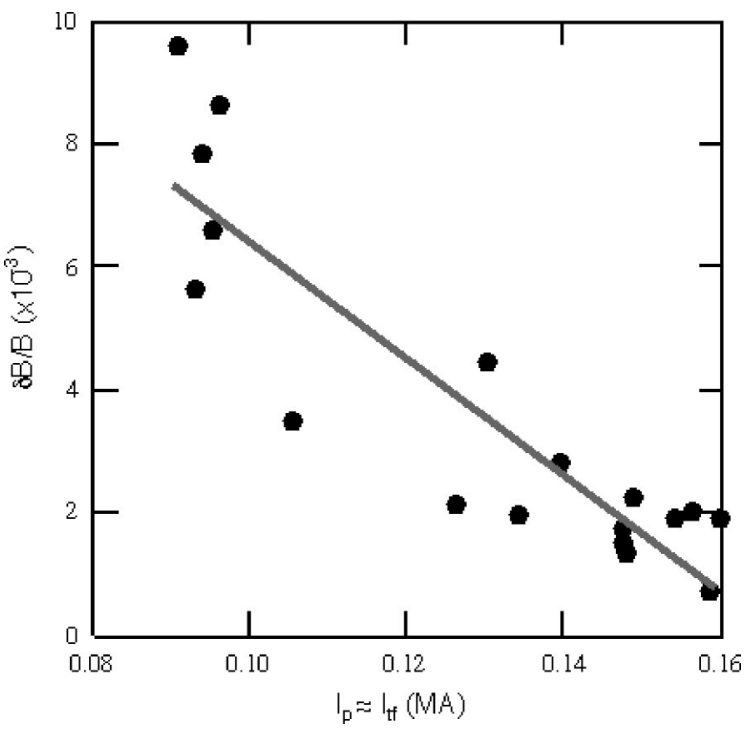

FIG. 10. Normalized MHD activity plotted vs toroidal field current along the $I_{p}=I_{t f}$ contour. A linear fit to the data is also shown.

safety factor profile at $t=16$ and $17 \mathrm{~ms}$. The mode activity is first observed at about $16 \mathrm{~ms}$, after the value of $q_{0}$ has crossed below 2. By $t=17 \mathrm{~ms}$, the $q=2$ surface has moved in toward the middle of the minor radius, where a resonant island could occupy a large volume of the plasma.
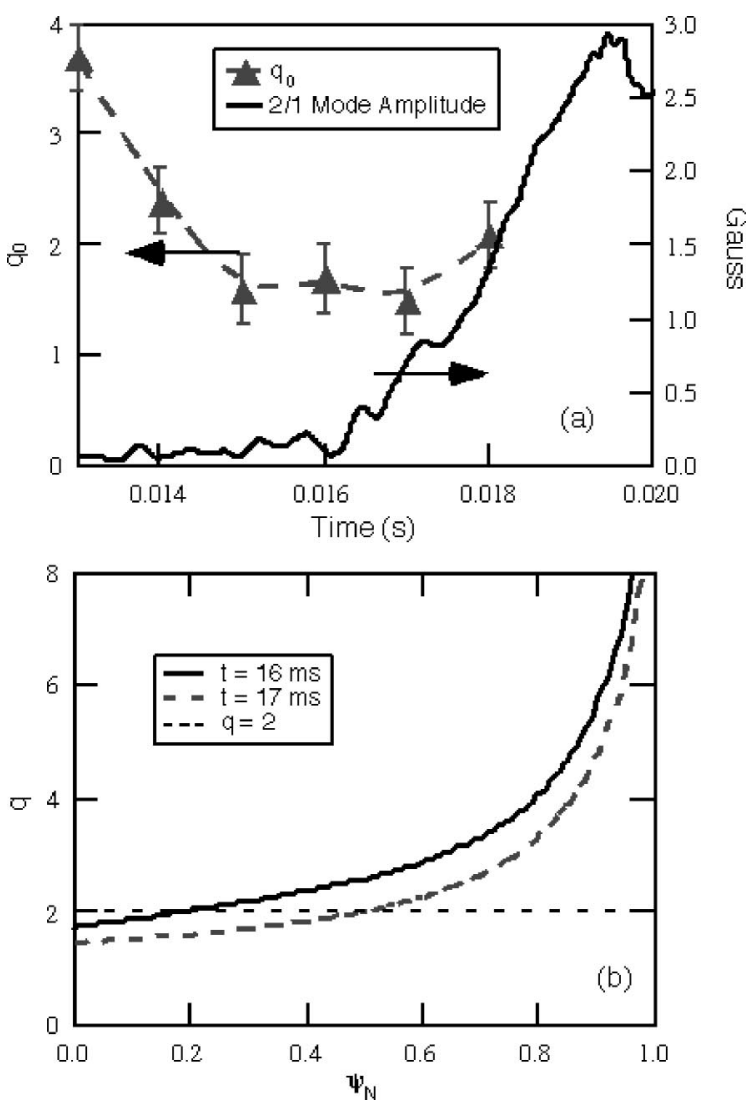

FIG. 11. Association of observed 2/1 mode activity with appearance of $q$ $=2$ surface in plasma: (a) $q_{0}$ vs time on left axis and mode amplitude vs time on right axis, and (b) safety factor profile at two times about the appearance of the mode. The $q=2$ level is also indicated.

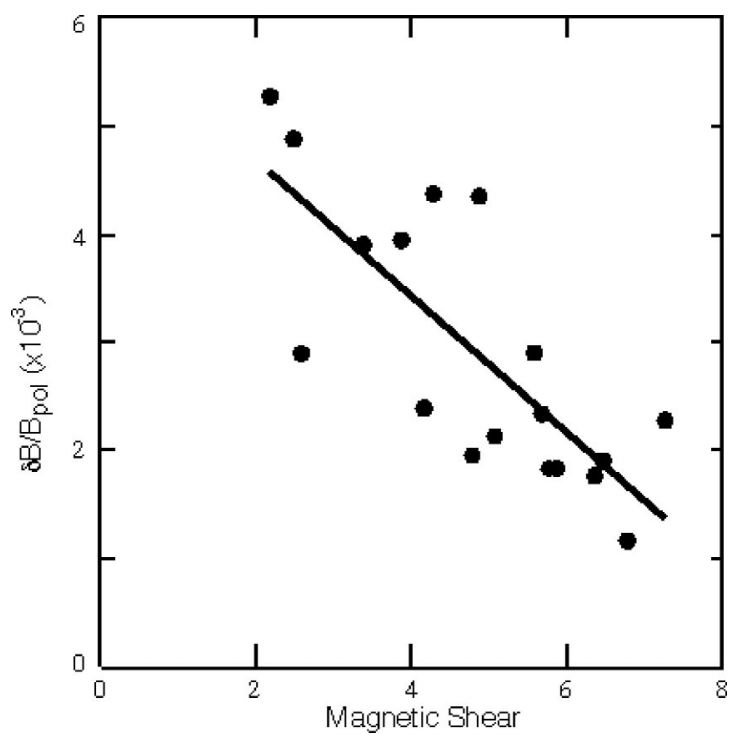

FIG. 12. Normalized MHD activity plotted vs magnetic shear at the relevant rational flux surface. A linear fit to the data is also shown.

The relation $I_{p}=I_{t f}$ essentially implies constant central safety factor, which can be seen from a simple approximation. For the central flux surfaces, the aspect ratio is relatively large and a simple expression for the value of $q$ can be posited:

$$
q(r)=\frac{2 \pi r^{2} B_{t}}{\mu_{0} R I(r)}\left[\frac{1+\kappa^{2}}{2}\right] .
$$

Making the assumption of a flat current profile implies

$$
q_{0} \approx \frac{I_{t f}\left(1+\kappa^{2}\right)}{I_{p}\left(2 A^{2}\right)} .
$$

For Pegasus at $I_{p}=I_{t f}$, Eq. (2) implies that $q_{0} \approx 1.5-2$, which is typically the observed range of values of $q_{0}$ at mode onset. At the time that $I_{p}=I_{t f}$, then, low-order rational flux surfaces are present in the plasma.

In particular optimized discharges the internal MHD activity is suppressed. These discharges achieve $I_{p}>I_{t f}$ and have higher stored energy than other plasmas with many similar conditions. It is found that these plasmas have relatively high magnetic shear and $q_{0}$ above 2 for several ms longer than other Pegasus discharges, as shown for a 0.15 MA plasma in Fig. 7. Figure 12 shows a plot of normalized MHD amplitude versus the magnetic shear at the relevant rational surface, indicating that the mode amplitude is inversely related to the local shear. These observations make it clear that the MHD activity can be suppressed both by increasing the safety factor in the low shear region of the plasma and by increasing the shear about the rational flux surfaces.

\section{B. External kink modes}

Some of the highest-current discharges produced in Pegasus are observed to terminate in abrupt disruptions. Analysis of these events indicates that these terminations are the result of edge kink modes associated with edge safety factor 

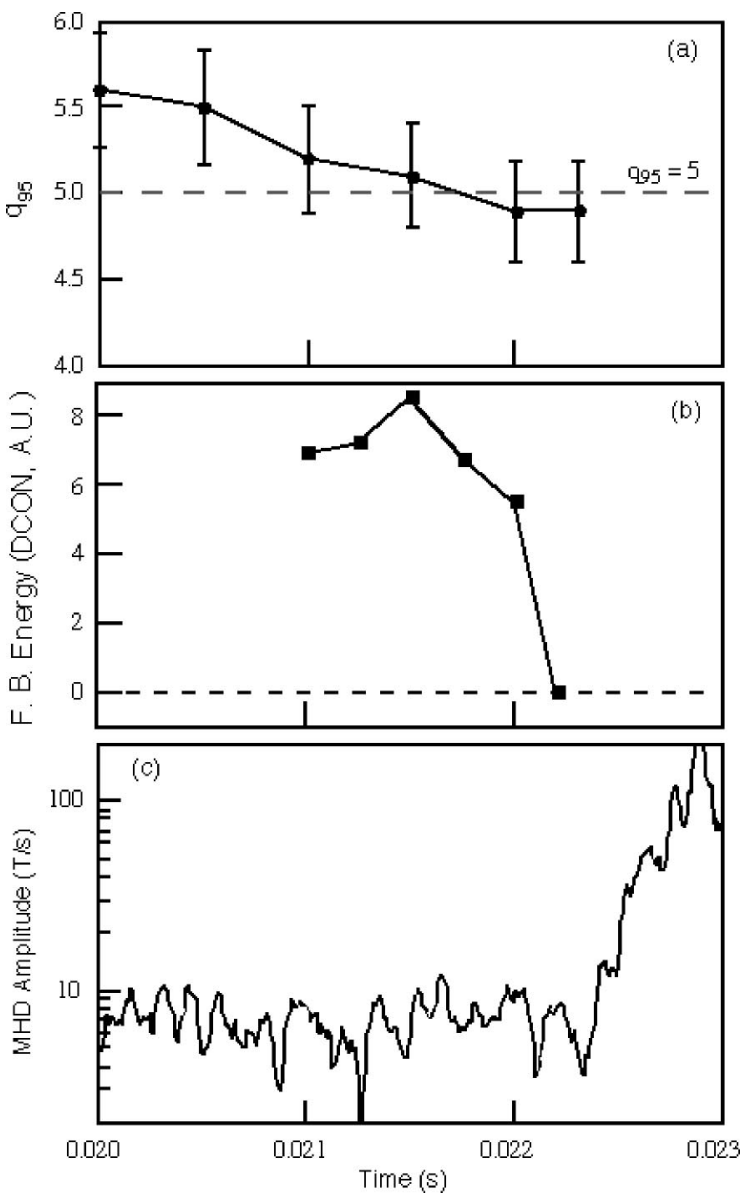

FIG. 13. External kink mode activity: (a) $q_{95}$ vs time along with $q_{95}=5$ line, (b) calculated free boundary energy from DCON, where negative energies indicate instability, and (c) amplitude of $n=1$ disruption precursor on a log scale.

$q_{95}$ crossing below 5, as illustrated in Fig. 13. The internal inductance $l_{i}$ is about 0.5 at this time. These modes are manifested by $n=1$ oscillations at roughly $10 \mathrm{kHz}$ observable on the high-field side toroidal Mirnov coil array. These oscillations grow on a time scale of approximately $90 \mu \mathrm{s}$, which is faster than the Alfvén time (a few $\mu \mathrm{s}$ ) but slower than the safety factor evolution time, $q_{95}\left(d q_{95} / d t\right)^{-1}$, which is approximately $20 \mathrm{~ms}$.

The DCON code ${ }^{18}$ was used to analyze the stability of these discharges to ideal MHD modes. Stability of the ideal kink is determined by the difference between the external vacuum magnetic energy and the total plasma energy; if this difference is negative, the plasma is unstable to the ideal kink. This difference is referred to as the "free boundary energy." Figure 13(b) shows the calculated free boundary energy for the discharge in Fig. 13(a) immediately before the disruption occurs. Figure 13(c) shows the amplitude of the observed core oscillations. It is seen that the calculated free boundary energy becomes negative just as the disruption precursor begins to grow, suggesting instability to the ideal kink. The observation of an ideal kink mode at $q_{95}=5$ is unusual by tokamak standards, where typically $q_{a}=2-3$ is required for instability to the ideal kink. This observation is in accordance with predictions ${ }^{3,4}$ that unstable values of $q_{95}$ increase as $A$ decreases. At the time of the instability the internal inductance is relatively low $\left(l_{i} \approx 0.5\right)$ and toroidal beta is considerable $\left(\beta_{t} \approx 18 \%\right)$. Both of these factors play a role in reducing stability to the external kink. The effect of these parameters has not yet been examined on Pegasus.

The results of this calculation are subject to uncertainties in the reconstruction due to measurement uncertainties. As stated above, the relative uncertainties in $q_{95}$ and $\beta_{t}$ are $6 \%$ and $15 \%$, respectively. Changes in these quantities within the error bars could qualitatively affect global stability. The effect of these errors on the calculated $n=1$ kink stability has not yet been comprehensively evaluated. However, multiple reconstructions produced by introducing random variations in the measurements on the order of their experimental uncertainties have generally indicated instability to the external kink for the discharge discussed in this section.

\section{SUMMARY AND FUTURE DIRECTIONS}

The Pegasus Toroidal Experiment is an ultralow aspect ratio toroidal device with the aim of characterizing plasma behavior and exploring stability limits with robust plasmas. In its initial phase of operation the experiment has demonstrated some of the important features of near-unity aspect ratio plasmas: toroidal beta up to $20 \%$ and normalized beta up to 5 with ohmic heating alone, normalized currents up to 6.5 , and significant paramagnetism (up to 50\%) and elongation (up to 3.7). The toroidal field utilization was found to reach a "soft limit" of unity. It was found that large tearing modes were responsible for this limit, with some reduction in available ohmic flux with reduced toroidal field also contributing. These tearing modes are present in almost all significant discharges due to the early presence of low-order rational flux surfaces in the plasma. Saturated island widths for these modes have been calculated to be large, greater than $0.1 \mathrm{~m}$, due to the low magnetic shear in the plasma center. These modes become more virulent as the toroidal field is lowered. Manipulation of the safety factor profile by increasing shear and $q_{0}$ during startup had the effect of mitigating the MHD activity and producing plasmas with $I_{p}$ exceeding $I_{t f}$. Pegasus has begun exploring the external kink stability boundary, finding instability at $q_{95}=5$ (for moderate internal inductance and finite beta), which is significantly larger than would be expected for a conventional tokamak.

Further exploration of limits in current and beta at nearunity aspect ratio will require the suppression of tearing modes early in the discharge and control over the external kink at low $I_{p} / I_{t f}$. These goals require improved control over the plasma. Specifically, improved control over the current ramp rate, evolution of the safety factor profile, radial position, and temperature during startup are required. Some control over $q_{95}$ is also highly desirable.

Upgrades being implemented on Pegasus during the fall and winter of 2002-2003 will provide these required control tools. All of the present LRC resonant power supplies are being replaced with capacitor banks switched actively by semiconductor switches such as integrated gate-commutated thyristors (IGCTs) arranged in an H-switch configuration to provide pulse-width modulation of the coil currents. In addi- 
tion, the toroidal field center rod will be replaced by a 12 turn, low inductance set. These upgrades will provide active control over the loop voltage and a 2.5 -fold increase in ohmic flux availability, a 3-fold increase in the toroidal field with fast rampdown capability, and significantly improved control over the plasma position and shape. In addition, divertor coils will be implemented both for divertor operation and for increased shape and safety factor control. These upgrades should allow improved access to stability limits due to higher beta and higher plasma current.

\section{ACKNOWLEDGMENTS}

The authors wish to thank the members of the Pegasus Team, especially Ben Ford and Greg Winz, for their efforts in building, maintaining, and operating the experiment. We also wish to thank the National High Magnetic Field Laboratory for building the ohmic solenoid, General Atomics for the engineering analysis of the vacuum vessel, Alan Glasser and Carl Sovinec for assistance with DCON, and Tony Peebles and his group at the University of California-Los Angeles for supplying the microwave interferometer. Helpful discussions with Alan Sykes, Mikhail Gryaznevich, and Martin Peng are gratefully acknowledged.
This work was supported by the United States Department of Energy Grant No. DE-FG02-96ER54375.

${ }^{1}$ Y-K. M. Peng and D. J. Strickler, Nucl. Fusion 26, 769 (1986).

${ }^{2}$ F. Troyon and R. Gruber, Phys. Lett. A 110, 29 (1985).

${ }^{3}$ A. D. Turnbull, F. Yasseen, A. Roy, O. Sauter, W. A. Cooper, S. Nicli, and F. Troyon, Nucl. Fusion 29, 629 (1989).

${ }^{4}$ A. Sykes, Phys. Plasmas 4, 1665 (1997).

${ }^{5}$ R. Fonck, G. Garstka, T. Intrator, B. Lewicki, T. Thorson, R. Toonen, K. L. Tritz, B. White, and G. Winz, Bull. Am. Phys. Soc. 41, 1400 (1996).

${ }^{6}$ D. A. Gates, R. Akers, L. Appel et al., Phys. Plasmas 5, 1775 (1998).

${ }^{7}$ M. Ono, M. G. Bell, R. E. Bigelow et al., Nucl. Fusion 41, 1435 (2001).

${ }^{8}$ A. Sykes, R. J. Akers, R. J. Appel et al., Nucl. Fusion 41, 1423 (2001).

${ }^{9}$ Y. Ono, M. Yamada, A. C. Jason, and F. M. Levinton, Phys. Fluids B 3, 1452 (1991).

${ }^{10}$ M. Yamada, N. Pomphrey, A. Morita, Y. Ono, and M. Katsurai, Nucl. Fusion 36, 1210 (1996).

${ }^{11}$ P. Pernambuco-Wise, B. L. Lesch, H. J. Schneider-Muntau, T. P. Intrator, R. J. Fonck, and G. R. Winz, Physica B 246-247, 350 (1998).

${ }^{12}$ L. Porte, C. L. Rettig, W. A. Pebbles, and X. Ngyuen, Rev. Sci. Instrum. 70, 1082 (1999)

${ }^{13}$ R. J. Fonck, A. T. Ramsey, and R. V. Yelle, Appl. Opt. 21, 2115 (1982).

${ }^{14}$ K. Tritz, R. Fonck, and T. Thorson, Rev. Sci. Instrum. 70, 595 (1999).

${ }^{15}$ A. Sykes, Plasma Phys. Controlled Fusion 43, A127 (2001).

${ }^{16}$ Z. Chang and J. D. Callen, Nucl. Fusion 30, 219 (1990).

${ }^{17}$ S. Ejima, R. W. Callis, J. L. Luxon, R. D. Stambaugh, T. S. Taylor, and J. C. Wesley, Nucl. Fusion 22, 1313 (1982).

${ }^{18}$ A. H. Glasser and M. S. Chance, Bull. Am. Phys. Soc. 42, 1848 (1997). 